\title{
Decreased Daily Melatonin Levels in Women with Systemic Lupus Erythematosus - A Short Report
}

Ralitsa Robeva1', Dobromir Tanev², Georgi Kirilov', Milena Stoycheva³ ${ }^{3}$ Analia Tomova², Philip Kumanov', Rasho Rashkov', Zlatimir Kolarov²

${ }^{1}$ Clinical Center of Endocrinology and Gerontology, Medical University Sofia, Medical Faculty, Sofia, Bulgaria

${ }^{2}$ Clinic of Rheumatology, Medical University Sofia, Medical Faculty, Sofia, Bulgaria

${ }^{3}$ Department of Social Medicine and Health Management, Medical University Sofia, Sofia, Bulgaria

\section{ABSTRACT}

Background: The neuroendocrine system is known to influence immunity, but the precise interactions between different hormones and autoimmune disorders remain obscure.

Aims: The present study aimed to investigate the role of daily serum melatonin concentrations in the development of systemic lupus erythematosus (SLE) in women.

Study Design: Case-control study

Methods: One-hundred and eleven SLE female patients and 46 healthy women were included in the study. Daily serum melatonin levels were investigated in all participants.

Results: SLE patients showed significantly lower daily melatonin levels in comparison to healthy women during the short photoperiod (17.75 \pm 7.13 $\mathrm{pg} / \mathrm{mL}$ [16.05] vs. $21.63 \pm 6.60 \mathrm{pg} / \mathrm{mL}$ [20.10], $\mathrm{p}=0.012$ ). Hormone concentrations were inversely related to the SLE activity index (SLEDAl) ( $r=-0.268$, $p=0.004$ ), but they did not correlate to any particular American College Rheumatology (ACR) criterion ( $>0.05$ for all).

Conclusion: Daily melatonin levels were decreased in women with systemic lupus erythematosus and correlated inversely to the activity of the autoimmune disease. Further studies are needed to clarify the importance of the pineal and extrapineal melatonin secretion in patients with systemic lupus erythematosus as well as the interrelations between hormones and autoimmunity.

Key Words: Melatonin, systemic lupus erythematosus, women, SLEDAI

Received: 04.11 .2012

Accepted: 29.04.2013

\section{Introduction}

Systemic lupus erythematosus (SLE) is a multisystem autoimmune disorder with a broad spectrum of clinical presentations that affects mostly women. No single cause for SLE has been identified (1). It is possible that the autoimmune disorder results from the combination of predisposing genetic factors and the disturbed status of stress response mechanisms, including the sympathetic nervous system and various hormones (2).

The neuroendocrine-immune system is regarded as a fundamental network supporting the health state that could play an important role in the development of autoimmune disorders. Key players of the neuroendocrine-immune pathways are steroid hormones as well as the neurohormone melatonin (3), which is considered a modulator of haematopoiesis and immune cell function. Melatonin can stimulate cytokine production, phagocytosis and natural killer cell activity; moreover, it can skew the immune response toward a helper T cell type 1 profile, whilst at the same time possibly acting as an anti-inflammatory agent (4). Therefore, melatonin concentrations have been investigated in different autoimmune, allergic and infectious diseases (5-7). Nevertheless, the complex role of the hormone on the immune system remains unclear. Considering the obscure data regarding the melatonin concentrations in SLE, we aimed to investigate the influence of the daily hormone levels on the development and clinical manifestations of lupus in women.

\section{Material and Methods}

One hundred and fifty-seven women aged between 19 and 67 years were included in the study. One hundred and eleven patients fulfilled the modified 1997 American College of Rheumatology (ACR) classification criteria for systemic lupus erythematosus: malar rash, discoid rash, photosensitivity, oral ulcer, arthritis, serositis, renal disorder, neurological disorder, haematological disorder, immunological disorder and antinuclear antibodies (8). All women underwent a complete general assessment. The SLE Disease Activity Index (SLEDAI) and the systemic lupus international collaborating clinics/ACR (SLICC) index were determined by one rheumatologist (9-11). SLEDAl represents the disease activity during the last 10 days prior to the assessment, while SLICC/ACR shows damages 
(non-reversible changes, not related to active inflammation) occurring since the onset of lupus, ascertained by clinical assessment and presented for at least 6 months $(10,11)$.

The previous and current medication with corticosteroids (intravenous methylprednisolone pulses as well as chronic peroral prednisone use), antimalarials and immunosuppressors were registered. Blood samples from the patients were collected immediately after the hospital entrance and prior to any subsequent pulse therapy. Previous pulse therapies were applied at least thirty days before the study. The cessation of chronic corticosteroid use was not possible because of ethical reasons.

Human melatonin rhythm could respond to changes in day length and, accordingly, different melatonin secretion patterns were described in long (April-September) and short photoperiods (October-March) $(12,13)$. Patients with SLE were recruited between August 2010 and January 2011, and were subsequently divided in two groups according to the month of blood sample collection: long photoperiod group (samples collected in August and September, $n=73$ ) and short photoperiod group (samples collected from October to January, $n=38$ ).

Forty six controls were collected from the medical staff and medical students. They were all clinically healthy women without any connective tissue diseases. All controls were recruited in the short photoperiod months (October - January).

The experimental protocol was explained to all participants and written informed consent was obtained. The study was carried out in accordance with the Declaration of Helsinki and approved by the institutional ethical committee.

\section{Hormone assay}

Blood samples were taken in the morning between 8 and $10 \mathrm{~h}$ and stored at $-20^{\circ} \mathrm{C}$ until assayed. Daily melatonin concentrations were investigated through Melatonin Direct RIA kits (DIAsource ImmunoAssay S.A., Nivelles, Belgium) with an analytical sensitivity of $2 \mathrm{pg} / \mathrm{mL}$, intra-assay variation of $9.8 \%$ and inter-assay variation of $9.6 \%$.

\section{Statistics}

All results were presented as mean $\pm \mathrm{SD}$ [median] for continuous variables or as a frequency (\%) for dichotomous variables. Categorical data were analysed through $\chi^{2}$ test or Fisher's exact test. Differences between the two groups were established using a Mann-Whitney or = independent t-test after a Kolmogorov-Smirnov test for normality of the distribution. Accordingly, two-tailed Spearman or Pearson correlations were calculated. All results were considered significant at the 0.05 level. Statistical analysis was conducted through SPSS v. 13 for Windows (SPSS, Chicago, IL, USA).

\section{Results}

The main characteristics of the investigated patients are shown in Table 1. The age did not differ significantly between the two groups $(41.90 \pm 12.01$ [41] vs. $43.83 \pm 12.29$ [44] years, $p=0.365)$. As expected, daily levels of melatonin in SLE women were significantly lower in the long photoperiod group than in the short photoperiod group $(13.59 \pm 8.06$ [12.30] vs.
$17.75 \pm 7.13 \mathrm{pg} / \mathrm{mL}$ [16.05], $\mathrm{p}=0.009)$. During the short photoperiod, daily pineal hormone levels were significantly lower in the lupus patients in comparison to the healthy controls (17.75 \pm 7.13 [16.05] vs. $21.63 \pm 6.60 \mathrm{pg} / \mathrm{mL}$ [20.10], $\mathrm{p}=0.012)$.

Melatonin concentrations were inversely related to the SLEDAI ( $r=-0.268, p=0.004)$, but not to the SLICC index (Figure 1). The relationships between melatonin and the activity of the disease remained significant after controlling for age, month of the sample collection and corticosteroid doses

Table 1. Main characteristics of the lupus patients

\begin{tabular}{|lc|}
\hline Characteristics & Women with SLE \\
\hline Age at diagnosis (years) & $34.63 \pm 11.58[34]$ \\
Duration of the disease (years) & $7.27 \pm 6.95[5]$ \\
ACR criteria & \\
Malar rash & $36.9 \%$ \\
Discoid rash & $10.8 \%$ \\
Photosensitivity & $72.1 \%$ \\
Oral ulcers & $23.4 \%$ \\
Arthritis & $99.1 \%$ \\
Serositis & $18.9 \%$ \\
Renal disorder & $23.4 \%$ \\
Neurological disorder & $30.6 \%$ \\
Haematological disorder & $64.0 \%$ \\
Immunological disorder & $78.4 \%$ \\
Antinuclear antibody & $71.2 \%$ \\
SLEDAl & $7.06 \pm 5.24[6]$ \\
SLICC & $0.80 \pm 1.05[1]$ \\
\hline ACR:American College & \\
\hline
\end{tabular}

ACR: American College of Rheumatology; SLEDAI: systemic lupus erythematodes disease activity index; SLICC: systemic lupus international collaborating clinics index

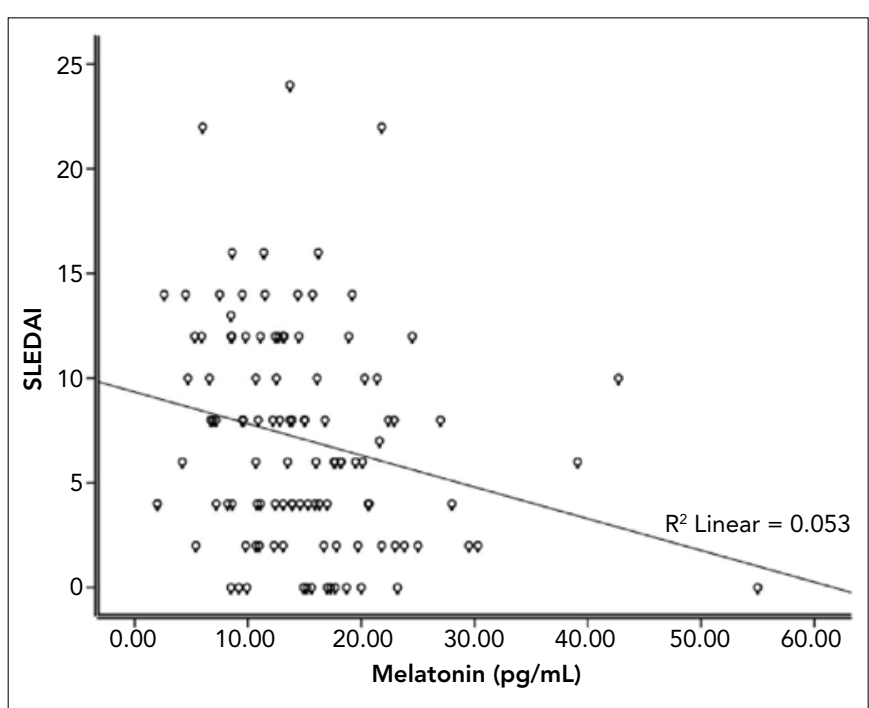

Figure 1. SLEDAl correlated with the melatonin concentrations in women with lupus 
$(r=-0.215, p=0.025)$. The melatonin levels were not significantly related to any particular ACR criterion, ACR criteria number, specific organ involvement or to immunological disturbances ( $p>0.05$ for all). Additionally, they were not significantly influenced by the chronic peroral treatment with antimalarials or immunosuppressors and did not correlate with chronic corticosteroid treatment doses ( $p>0.05$ ).

In order to reveal the significance of the lowest melatonin levels, the presence of different organic involvement was compared between the patients with melatonin levels below the $25^{\text {th }}$ percentile and other patients. The melatonin decrease was not related to the development of renal impairment, cardiac or pulmonary disturbances, serositis, vasculitis or haematological disorders $(p>0.05)$. However, neurological disorders tended to be more frequent in the group of SLE patients with the lowest melatonin levels $(41.0 \%$ vs. $25.0 \%, p=0.089)$.

\section{Discussion}

The present study showed significantly lower daily melatonin levels in women with SLE compared to healthy women. However, in MRL/MP-fas lupus-prone mice, a different pattern, uncoupled from the light/dark cycle secretion, with paradoxically high melatonin levels during the light phase has been described (14). Since nocturnal rodents and daily active humans have very different visual and circadian systems as well as quite different responses to light, the observations of mice lupus models could not be directly transferred to SLE patients (15). To the best of our knowledge, this is the first study comparing melatonin levels in healthy women and SLE patients; however, a case report of a patient with SLE and a pineal teratoma with a subsequent malignant transformation has been described (16). Therefore, further studies are needed to confirm or reject our results.

The decreased melatonin levels in women with lupus could be a consequence of the chronic autoimmune process or immunosuppressive treatment. Immune complex deposits in the pineal gland were observed in an experimental model of SLE (17). Thus, the decreased pineal secretion could be a sign of neurolupus. The higher prevalence of neurological impairment in patients with the lowest melatonin levels supported this assumption. However, a protective role of the increased melatonin concentrations on the brain could not be excluded, considering the known antioxidant features of the hormone (18).

Exogenous corticosteroids were related to both increased and decreased nocturnal melatonin levels in healthy humans $(19,20)$. Increased daily melatonin levels were found in female Cushing patients irrespective of the cause, which returned to normal after the hypercortisolism correction (21). Probably, the decrease of the daily melatonin levels in SLE patients was not a consequence of the chronic corticosteroid treatment. In accordance, no relationships between melatonin levels and corticosteroid doses were established.

Melatonin concentrations were not only decreased in lupus patients but they also correlated inversely with the SLEDAI index of disease activity. Several investigations either directly or indirectly supported the relationships between the hormone and the autoimmune process. The administration of melatonin in female lupus MRL/MP-fas mice reduced the levels of autoantibodies and improved the histological changes (22). Since the hormone was added to the drinking water, the exact time of melatonin application could not be specified in the latter study, but it could be of clinical importance. For instance, the daily melatonin administration did not significantly enhance the survival of lupus NZB/W mice when injections were performed between 17:00 and 19:00h but did enhance survival when given between 08:00 and 10:00h (23). These studies suggested that, at least in female mice models of spontaneous lupus, the melatonin use was beneficial.

The first and only study of melatonin in humans with lupus was conducted by Haga et al. (24). The authors examined seasonal variations in SLE activity as well as daily melatonin levels in lupus patients living in a subarctic region. The daily melatonin plasma levels in 14 patients with identical treatment regimen tended to be higher in December than in June. They did not correlate significantly with SLEDAl, arthritis, flare, C-reactive protein, anti-DNA or anti-nuclear antibodies in June and December, but correlated inversely with erythrocyte sedimentation rate (ESR) in December. The authors concluded that melatonin levels were not related to the clinical disease activity or manifestation, although the correlation with ESR may indicate some connection to SLE disease activity (24). Our study supported the latter assumption, although the two studies were difficult to compare: the Norwegian patients were recruited in the subarctic region with minimal winter sunlight, while our study was conducted in a region with a moderate climate and 80-100 average monthly hours of sunshine during the winter (25).

Circadian and circannual fluctuations of the melatonin concentrations depend on the pineal melatonin formation, while basal daytime hormone levels might be additionally controlled by other factors (26). For instance, the neuroendocrine cells of the gastrointestinal tract could significantly affect the daytime circulating concentrations of melatonin (27). A study in pineal melatonin deficient mice indicated that different immune cells and tissues were able to synthesise melatonin; thus, they could contribute to the detectable melatonin levels in these animals (28). In the present study, only daily melatonin concentrations were determined, as in other studies $(24,29)$. Therefore, no information about the possible disturbances in the circadian melatonin rhythm was obtained, which was considered the main limitation of the study. Another important limitation was the lack of longitudinal data allowing the follow up of the individual melatonin changes during the SLE progression. Nevertheless, our results showed significant interrelations between the daily melatonin concentrations and SLE activity. Further studies are needed to clarify the importance of the pineal and extrapineal melatonin secretion in patients with systemic lupus erythematosus as well as the interrelations between the hormone and the autoimmunity.

Ethics Committee Approval: Ethics committee approval was received for this study. 
Informed Consent: Written informed consent was received from the participants of this study.

Peer-review: Externally peer-reviewed.

Author contributions: Concept - R.R., D.T., Z.K., R.R., P.K.,; Design R.R., D.T., Z.K., R.R., M.S.,P.K.; Supervision - G.K., A.T., P.K., R.R., Z.K.; Resource - Z.K., R.R., G.K.; Materials - D.T., Z.K., R.R.; Data Collection\&/or Processing - D.T., R.R. A.T., M.S., G.K.; Analysis\&/or Interpretation - R.R., D.T., M.S., A.T.; Literature Search - R.R., D.T.; Writing - R.R., D.T., M.S.; Critical Reviews - G.K., A.T., P.K., R.R., Z.K.

Conflict of Interest: No conflict of interest was declared by the authors.

Financial Disclosure: This study was financially supported by the Medical University Sofia (Grant No: 26/2010, Grant No: 59/2011).

\section{References}

1. Cervera R, Espinosa G, D'Cruz D. Systemic lupus erythematosus: pathogenesis, clinical manifestations and diagnosis. In: Bijlsma JW, editor. EULAR Compendium on Rheumatic Diseases. BMJ Publishing Group and European League Against Rheumatism; 2009,p.257-68.

2. Cutolo $M$, Capellino $S$, Straub R. Sex hormones, the immune system and autoimmune diseases. In: Asherson R, editor. Handbook of Systemic Autoimmune Diseases vol. 9. Walker S, Jara L, editors. Endocrine manifestations of systemic autoimmune diseases, Oxford, Elsevier; 2008, p. 13-19.

3. Cutolo M, Straub RH. Insights into endocrine-immunological disturbances in autoimmunity and their impact on treatment. Arthritis Res Ther 2009;2:218. [CrossRef]

4. Szczepanik M. Melatonin and its influence on immune system. J Physiol Pharmacol 2007;58:115-24.

5. Sulli A, Maestroni GJ, Villaggio B, Hertens E, Craviotto C, Pizzorni $\mathrm{C}$, et al. Melatonin serum levels in rheumatoid arthritis. Ann N Y Acad Sci 2002;966:276-83. [CrossRef]

6. Fei GH, Liu RY, Zhang ZH, Zhou JN. Alterations in circadian rhythms of melatonin and cortisol in patients with bronchial asthma. Acta Pharmacol Sin 2004;25:651-56.

7. Carrillo-Vico A, Lardone PJ, Naji L, Fernández-Santos JM, MartínLacave I, Guerrero JM, et al. Beneficial pleiotropic actions of melatonin in an experimental model of septic shock in mice: regulation of pro-/anti-inflammatory cytokine network, protection against oxidative damage and anti-apoptotic effects. J Pineal Res 2005;39:400-08. [CrossRef]

8. Hochberg MC. Updating the American College of Rheumatology revised criteria for classification of systemic lupus erythematosus. Arthritis Rheum 1997;40:1725. [CrossRef]

9. Stoll T, Seifert B, Isenberg DA. SLICC/ACR Damage Index is valid, and renal and pulmonary organ scores are predictors of severe outcome in patients with systemic lupus erythematosus. Br J Rheumatol 1996;35:248-54. [CrossRef]

10. Gladman D, Ginzler E, Goldsmith C, Fortin P, Liang M, Urowitz $M$, et al. The development and initial validation of the Systemic Lupus International Collaborating Clinics/American College of Rheumatology damage index for systemic lupus erythematosus. Arthritis Rheum 1996;39:363-9. [CrossRef]

11. Bombardier C, Gladman DD, Urowitz MB, Caron D, Chang CH. Derivation of the SLEDAI. A disease activity index for lupus patients. The Committee on Prognosis Studies in SLE. Arthritis Rheum 1992;35:630-40. [CrossRef]
12. Wehr TA. The durations of human melatonin secretion and sleep respond to changes in daylength (photoperiod). J Clin Endocrinol Metab 1991;73:1276-80. [CrossRef]

13. Luboshitzky R, Yanai D, Shen-Orr Z, Israeli E, Herer P, Lavie P. Daily and seasonal variations in the concentration of melatonin in the human pineal gland. Brain Res Bull 1998;47:271-6. [CrossRef]

14. Lechner $O$, Dietrich $H$, Oliveira dos Santos A, Wiegers GJ, Schwarz S, Harbutz $M$, et al. Altered circadian rhythms of the stress hormone and melatonin response in lupus-prone MRL/MPfas(lpr) mice. J Autoimmun 2000;14:325-33. [CrossRef]

15. Bullough JD, Rea MS, Figueiro MG. Of mice and women: light as a circadian stimulus in breast cancer research. Cancer Causes Control 2006;17:375-83. [CrossRef]

16. Shah A, Sadasivan B, Leng BM, Ho J. A teratocarcinoma developing after gross total excision of a pineal teratoma in a patient with systemic lupus erythematosus. Neurol Res 1995;17:229-32.

17. Peress NS, Perillo E, Fenstermacher JD. Circumventricular organs in chronic serum sickness: a model for cerebral lupus. Biol Psychiatry 1989;26:397-07. [CrossRef]

18. Reiter RJ, Tan DX, Allegra M. Melatonin: reducing molecular pathology and dysfunction due to free radicals and associated reactants. Neuro Endocrinol Lett 2002;23:3-8.

19. Lang U, Theintz G, Rivest RW, Sizonenko PC. Nocturnal urinary melatonin excretion and plasma cortisol levels in children and adolescents after a single oral dose of dexamethasone. Clin Endocrinol (Oxf) 1986;25:165-72. [CrossRef]

20. Demisch L, Demisch K, Nickelsen T. Influence of dexamethasone on nocturnal melatonin production in healthy adult subjects. $J$ Pineal Res 1988;5:317-22. [CrossRef]

21. Tomova A, Kumanov P, Robeva R, Manchev S, Konakchieva R. Melatonin secretion and non-specific immune responses are differentially expressed in corticotropin-dependent and corticotropin-independent Cushing's syndrome. Med Sci Monit 2008;14:327-32

22. Jimenez-Caliani AJ, Jimenez-Jorge $S$, Molinero P, Fernandez-Santos JM, Martin-Lacave I, Rubio A, et al. Sex-dependent effect of melatonin on systemic erythematosus lupus developed in $\mathrm{Mrl} / \mathrm{Mpj}$-Faslpr mice: it ameliorates the disease course in females, whereas it exacerbates it in males. Endocrinology 2006;147:1717-24. [CrossRef]

23. Lenz SP, Izui S, Benediktsson H, Hart DA. Lithium chloride enhances survival of NZB/W lupus mice: influence of melatonin and timing of treatment. Int J Immunopharmacol 1995;17:581-92. [CrossRef]

24. Haga HJ, Brun JG, Rekvig OP, Wetterberg L. Seasonal variations in activity of systemic lupus erythematosus in a subarctic region. Lupus 1999;8:269-73. [CrossRef]

25. World weather and climate information. http://www.weatherand-climate.com, data access 17.01.2013.

26. Huether $\mathrm{G}$. The contribution of extrapineal sites of melatonin synthesis to circulating melatonin levels in higher vertebrates. Experientia 1993;49:665-70. [CrossRef]

27. Bubenik GA. Gastrointestinal melatonin: localization, function, and clinical relevance. Dig Dis Sci 2002;47:2336-48. [CrossRef]

28. Gómez-Corvera A, Cerrillo I, Molinero P, Naranjo MC, Lardone PJ, Sanchez-Hidalgo $M$, et al. Evidence of immune system melatonin production by two pineal melatonin deficient mice, C57BL/6 and Swiss strains. J Pineal Res 2009;47:15-2. [CrossRef]

29. Caglayan S, Ozata M, Ozisik G, Turan M, Bolu E, Oktenli C, et al. Plasma melatonin concentration before and during testosterone replacement in Klinefelter's syndrome: relation to hepatic indolamine metabolism and sympathoadrenal activity. J Clin Endocrinol Metab 2001;86:738-43. [CrossRef] 